Org Lett. 2016 July 01; 18(13): 3286-3289. doi:10.1021/acs.orglett.6b01580.

\title{
A Modular, Catalytic Enantioselective Construction of Quaternary Carbon Stereocenters by Sequential Cross-Coupling Reactions
}

\author{
Bowman Potter, Emma K. Edelstein, and James P. Morken ${ }^{\star}$ \\ Department of Chemistry, Merkert Chemistry Center, Boston College, Chestnut Hill, \\ Massachusetts 02467
}

\begin{abstract}
The catalytic Suzuki-Miyaura cross-coupling with chiral $\gamma, \gamma$-disubstituted allylboronates in the presence of RuPhos ligand occurs with high regioselectivity and enantiospecificity, furnishing nonracemic compounds with quaternary centers. Mechanistic experiments suggest that the reaction occurs by transmetallation with allyl migration, followed by rapid reductive elimination.
\end{abstract}

\section{Graphical Abstract}

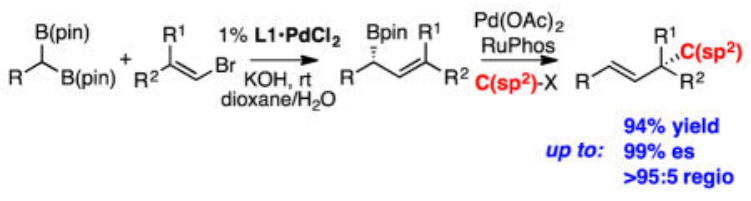

The selective and efficient construction of quaternary carbon stereocenters remains a challenging task for asymmetric catalysis. ${ }^{1}$ While several advances have been charted, one method that has not been broadly employed for this undertaking is the Suzuki-Miyaura cross-coupling reaction. ${ }^{2} \mathrm{~A}$ lack of advances in this area can plausibly be traced to the expectation that such catalytic reactions would require the intermediacy of highly hindered tertiary organopalladium complexes, and access to these compounds might be prohibitive. Indeed, aside from asymmetric catalytic allyl-allyl cross-coupling, ${ }^{3}$ the combination of an organoboron reagent and an organic electrophile to furnish a nonracemic quaternary carbon stereocenter selectively, has yet to be been accomplished. ${ }^{4}$ In this manuscript, we describe a site-selective cross-coupling of allylboronates that, when conducted in sequence with enantiotopic-group-selective cross-coupling, enables a modular assembly of enantiomerically-enriched quaternary carbon centers from geminal bis(boronates), alkenyl halides, and $\mathrm{C}\left(\mathrm{sp}^{2}\right)$ electrophiles (Scheme 1).

*Corresponding Author: morken@bc.edu. Author Contributions

The manuscript was written through contributions of all authors. All authors have given approval to the final version of the manuscript. The authors declare no competing financial interest.

Supporting Information. Procedures, characterization and spectral data. This material is available free of charge via the Internet at http://pubs.acs.org. 
With respect to the development of the sequence represented in Scheme 1, we recently established an enantiotopic-group-selective cross coupling to construct requisite $\gamma, \gamma^{\prime}$ disubstituted allylboronates. ${ }^{5}$ However, the utility of these reagents in asymmetric crosscoupling reactions has little precedent. Important recent studies in the cross-coupling of allylboron reagents and $\mathrm{C}\left(\mathrm{sp}^{2}\right)$ electrophiles have shed light on critical aspects of regioselectivity in $\mathrm{C}-\mathrm{C}$ bond formation. Moderate branch-selectivity in cross-coupling of crotyl boronates and aryl electrophiles was first observed by Kalinin. ${ }^{6}$ This reaction was developed more broadly by Miyaura, who found that these reactions could be accomplished enantioselectively with chiral catalysts, and that they could exhibit very high regioselectivity for the $\gamma$-coupled products ostensibly by $\mathrm{S}_{\mathrm{E}} 2^{\prime}$ transmetallation followed by rapid reductive elimination. ${ }^{7}$ Our own group recently disclosed an enantioselective, intra-molecular allylaryl cross-coupling to furnish carbocycles with high levels of selectivity. ${ }^{8}$ With more hindered $\gamma, \gamma$-disubstituted reagents, Organ found that Pd-PEPPSI complexes catalyze linear-selective prenylation of electrophiles, ${ }^{9}$ while Buchwald observed that with appropriate choice of phosphine ligand, either the linear adduct or the branched tert-prenyl crosscoupling product could be obtained selectively. ${ }^{10}$ Studies by Crudden and Aggarwal, ${ }^{11}$ and more recently by Hall, ${ }^{12}$ determined that cross-coupling of secondary allylic boronates could furnish tertiary stereocenters, and that such reactions occur with enantiospecificity. However, to our knowledge the issue of chirality transfer in the generation of quaternary centers has yet to be addressed.

With the goal of developing a cross-coupling reaction sequence that might be accomplished in a single flask process, our studies commenced by examining the reaction between nonracemic allylboronate $\mathbf{1}$ and aromatic electrophiles (Scheme 2). A critical criteria for the eventual development of a tandem single flask process was to employ the same conditions for the allyl-aryl cross coupling that were employed for the enantiotopic-group-selective coupling in Scheme $1\left(\mathrm{KOH} /\right.$ dioxane/ $\left.\mathrm{H}_{2} \mathrm{O}\right)$. In the final rendition, it was envisaged that one need not isolate the intermediate allylboronate prior to its engagement in a second crosscoupling. As depicted in Table 1, it was found that efficient, site selective, and highly enantiospecific cross-couplings could be obtained by employing $\mathrm{Pd}(\mathrm{OAc})_{2}$ in the presence of RuPhos ${ }^{13}$ ligand. With this catalyst combination and reaction conditions, efficient reactions and very high levels of $\gamma: a$ cross-coupling products were observed. Moreover, the reaction products were isolated with $>20: 1 \mathrm{E}: Z$ olefin stereoiomer ratios and with excellent levels of enantiospecificity (generally $>95 \%$ es). As the examples in Scheme 2 indicate, the reaction appears to be effective with both electron-rich and electron poor aryl bromides as the electrophile. It was also found that aryl chlorides and iodides participated with similar efficiency: compound $\mathbf{8}$ could be accessed from 4-chloroanisole or 4-iodoanisole in $95 \%$ yield and $88 \%$ yield respectively, and in identical selectivity as with the aryl bromide electrophile. In addition to effective couplings with aryl electrophiles, heteroaromatic compounds also appear to participate in the reaction (products 13-17), and products 18-24 demonstrate that alkenyl electrophiles engage in stereo-specific cross-couplings and provide a route to important 1,4 "skipped dienes"14 that bear a quaternary carbon stereocenter. Also of note, products $\mathbf{2 6}-\mathbf{2 8}$ suggest that versatile aryl chloride, alkene, and silyl ether functional groups can be accommodated in the allylboronate partner. Lastly, while the data in Scheme 2 is for reactions conducted on $0.1 \mathrm{mmol}$ scale, when the synthesis of 9 was conducted on 2 
mmol scale, the reaction proceeded with equal efficiency and selectivity (91\% yield, > $95: 5$ $\gamma: a, 99 \%$ es).

The high level of stereospecificity in the $\mathrm{Pd} / \mathrm{RuPhos}$ catalyzed allyl-aryl cross-coupling suggests that judicious choice of substrates can allow the same enantiomer of chiral ligand to furnish both enantiomers of chiral cross-coupling product. This feature is demonstrated in Scheme 3, where it is shown that cross-coupling of boronate 29 with either $E$ or $Z$-1bromo-2-methyl-1-butene ${ }^{15}$ can furnish diastereomeric cross-coupling products $\mathbf{3 0}$ and $\mathbf{3 1}$ in near equal levels of stereoselection. Subjection of $\mathbf{3 0}$ and $\mathbf{3 1}$ to catalytic allyl-aryl crosscoupling furnishes enantiomeric products $(R)$-32 and $(S)$-32, both in high levels of enantiomeric purity. The absolute configuration of the enantiomers of $\mathbf{3 2}$ was established by ozonolysis and oxidation (Jones reagent) to the derived carboxylic acid followed by comparison of optical rotation to that for the known compound. ${ }^{16}$

With an effective cross-coupling that delivers the $\gamma$-coupling product selectively from $\gamma, \gamma$ disubstituted allylboronates, single-flask cascade cross-coupling sequences were investigated. First, to determine whether residual $\mathbf{L} \mathbf{1} \cdot \mathbf{P d C l} \mathbf{l}_{\mathbf{2}}$ catalyst used for the crosscoupling of geminal bis(boronates) would undermine the subsequent enantiospecific allylaryl cross-coupling reaction, we probed whether $\mathbf{L 1} \cdot \mathbf{P d C l} \mathbf{l}_{\mathbf{2}}$ would effect cross-coupling of bromobenzene and in situ generated allylboronate. As depicted in eq 1 (Scheme 4), heating with $\mathrm{PhBr}$ at the end of a cross-coupling between geminal bis(boronate) 29 and alkenyl bromide 33 resulted low conversion of allylboronate $\mathbf{3 4}$ thus $\mathbf{L 1} \cdot \mathbf{P d C l}_{\mathbf{2}}$ appears to be ineffective at allyl-aryl cross-coupling. To probe the capacity for a single-flask cascade sequence, 29 and 33 were then subjected to cross-coupling with $\mathbf{L 1} \cdot \mathbf{P d C l}_{2}$ with $\mathrm{KOH}$ in dioxane/water, then 4-chloroanisole was added followed by $\mathrm{Pd}(\mathrm{OAc})_{2}$ and RuPhos. Upon heating to $60{ }^{\circ} \mathrm{C}$ for an additional 14 hours, coupling product 8 was isolated in $64 \%$ yield and 93:7 er (eq 2). A similar single-flask reaction sequence furnished $\mathbf{2 2}$ with comparable yield and selectivity.

We considered that an intriguing example of the utility of the cascade cross-coupling sequence to deliver quaternary compounds might lie in the construction of nonracemic $\mathbf{3 8}$ (Scheme 5), the simplest all-carbon quaternary-center-containing compound. ${ }^{17}$ This compound has recently been addressed by Aggarwal ${ }^{18}$ and Cramer $^{19}$ through approaches employing asymmetric homologation and selective cleavage of enantiotopic $\mathrm{C}-\mathrm{C}$ bonds, respectively. As depicted in Scheme 5, 38 was readily prepared in a three-step sequence involving asymmetric cross-coupling of bis(boronate) $\mathbf{3 5}$ with alkenyl halide $\mathbf{3 6}$, to give $\mathbf{3 7}$. Subsequent stereospecific cross-coupling of $\mathbf{3 7}$ with vinyl bromide followed catalytic hydrogenation furnished the target hydrocarbon $\mathbf{3 8}$ in $72 \%$ yield as determined by ${ }^{13} \mathrm{C}$ NMR versus an internal standard.

In terms of reaction mechanism, one of two reasons could account for the $\gamma$-selectivity in the RuPhos/Pd catalyzed allyl-aryl cross-coupling reaction. In line with studies by Buchwald ${ }^{10}$ on the construction of quaternary carbons by Pd-catalyzed coupling of allylboronates and related studies by Aggarwal and Cruuden ${ }^{11 b}$, it could be that stereospecific $\mathrm{S}_{\mathrm{E}} 2^{\prime}$ transmetallation is followed by rapid reductive elimination; alternatively, the transmetallation (either $\mathrm{S}_{\mathrm{E}} 2^{\prime}$ or $\mathrm{S}_{\mathrm{E}}$ ) generates a $\pi$-allyl complex that favors reductive 
elimination at the more substituted carbon. To probe these possibilities, substituted secondary allylboronate 39 was subjected to $\mathrm{Pd} / \mathrm{RuPhos}$ catalyzed cross-coupling and found to react with $>98 \% \gamma$-selectivity to give $\mathbf{4 0}$ (eq 4, Scheme 6); in contrast, when regioisomeric tertiary allylboronate $\mathbf{4 1}$ was subjected to the reaction conditions, regioisomer 42 was produced in excellent selectivity (eq. 5). This pair of observations effectively rules out the possibility for $\pi$-allyl isomerization of (allyl)palladium intermediates. Collectively, the site-selectivity of the reaction, the configuration of the product alkene, and the correlation between allylboronate configuration and product configuration is most in line with an inner-sphere $s y n-\mathrm{S}_{\mathrm{E}} 2^{\prime}$ transmetallation that operates by structure A below (eq 6 , Scheme 6).

In summary, we have reported a stereospecific cross-coupling reaction that applies to highly substituted allylboronates and furnishes quaternary stereocenters with outstanding stereoselection. Along with enantiotopic-group-selective cross-coupling, the allyl-aryl crosscoupling can be run as a part of a two step sequence to create useful chiral hydrocarbons directly from simple precursors.

\section{Supplementary Material}

Refer to Web version on PubMed Central for supplementary material.

\section{Acknowledgments}

The NIH (GM-64451) is acknowledged for financial support; B. P. is grateful for a LaMattina graduate fellowship.

\section{References}

1. For recent reviews: Das JP, Marek I. Chem Commun. 2011; 47:4593.Hong AY, Stolz BM. Eur J Org Chem. 2013:2745.Quasdorf KW, Overman LE. Nature. 2014; 516:181. [PubMed: 25503231] Marek I, Minko Y, Pasco M, Mejuch T, Gilboa N, Chechik H, Das JP. J Am Chem Soc. 2014; 136:2682. [PubMed: 24512113] Eppe G, Didier D, Marek I. Chem Rev. 2015; 115:9175. [PubMed: 25768205]

2. For a review of stereospecific cross-coupling, see: Cherney AH, Kadunce NT, Reisman SE. Chem Rev. 2015; 115:9587. [PubMed: 26268813]

3. (a) Zhang P, Le H, Kyne RE, Morken JP. J Am Chem Soc. 2011; 133:9716. [PubMed: 21648464]

(b) Ardolino MJ, Morken JP. J Am Chem Soc. 2014; 136:7092. [PubMed: 24720611]

4. For an alternative synthesis of nonracemic asymmetric quaternary carbon centers from organoboronates, see: Bonet A, Odachowski M, Leonori D, Essafi S, Aggarwal VK. Nat Chem. 2014; 6:584. [PubMed: 24950327] Llaveria J, Leonori D, Aggarwal VK. J Am Chem Soc. 2015; 137:10958. [PubMed: 26292943]

5. (a) Potter B, Szymaniak AA, Edelstein EK, Morken JP. J Am Chem Soc. 2014; 136:17918. [PubMed: 25482206] (b) Sun C, Potter B, Morken JP. J Am Chem Soc. 2014; 136:6534. [PubMed: 24564423]

6. Kalinin VN, Denisov FS, Bubnov YN. Mendeleev Commun. 1996; 6:206.

7. (a) Yamamoto Y, Takada S, Miyaura N. Chem Lett. 2006; 35:1368.(b) Yamamoto Y, Takada S, Miyaura N, Iyama T, Tachikawa H. Organometallics. 2009; 28:152.

8. Schuster CH, Coombs JR, Kasun ZA, Morken JP. Org Lett. 2014; 16:4420. [PubMed: 25105510]

9. Farmer JL, Hunter HN, Organ MG. J Am Chem Soc. 2012; 134:17470. [PubMed: 23046477]

10. Yang Y, Buchwald SL. J Am Chem Soc. 2013; 135:10642. [PubMed: 23837686] 
11. (a) Glasspoole BW, Ghozati K, Moir JW, Crudden CM. Chem Commun. 2012; 48:1230.(b) Chausset-Boissarie L, Ghozati K, LaBine E, Chen JL-Y, Aggarwal VK, Crudden CM. Chem Eur J. 2013; 19:17698. [PubMed: 24302640]

12. Rybak T, Hall DG. Org Lett. 2015; 17:4156. [PubMed: 26291472]

13. Charles MD, Schultz P, Buchwald SL. Org Lett. 2005; 7:3965. [PubMed: 16119943] . For crosscoupling alkylB(pin) reagents with aryl halides, see: Yang CT, Zhang ZQ, Tajuddin H, Wu CC, Liang J, Liu JH, Fu Y, Czyzewska M, Steel PG, Marder TB, Liu L. Angew Chem Int Ed. 2012; 124:543.

14. Jie M, Pasha MK, Syed-Rahmatullah MSK. Nat Prod Rep. 1997; 14:163.

15. Lim S, Wipf P. Angew Chem Int Ed Engl. 1993; 32:1068.

16. Ruano JLG, Martin-Castro AM, Tato F, Torrente E, Poveda AM. Chem Eur J. 2010; 16:6317. [PubMed: 20411540]

17. (a) Hoeve WT, Wynberg H. J Org Chem. 1980; 45:2754.(b) Fujita T, Obata K, Kuwahara S, Miura N, Nakahashi A, Monde K, Decatur J, Harada N. Tetrahedron Lett. 2007; 48:4219.(c) Fujita T, Obata K, Kuwahara S, Nakahashi A, Monde K, Decatur J, Harada N. Eur J Org Chem. 2010:6372. (d) Simaan S, Goldberg AFG, Rosset S, Marek I. Chem Eur J. 2010; 16:774. [PubMed: 19950341]

18. Pulis AP, Blair DJ, Torres E, Aggarwal VK. J Am Chem Soc. 2013; 135:16054. [PubMed: 24138162]

19. Seiser T, Cramer N. J Am Chem Soc. 2010; 132:5340. [PubMed: 20345153] 


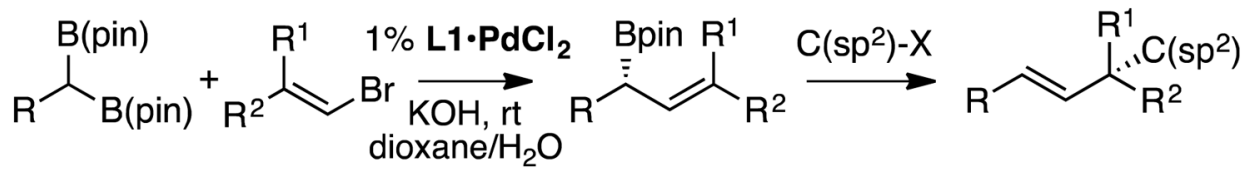

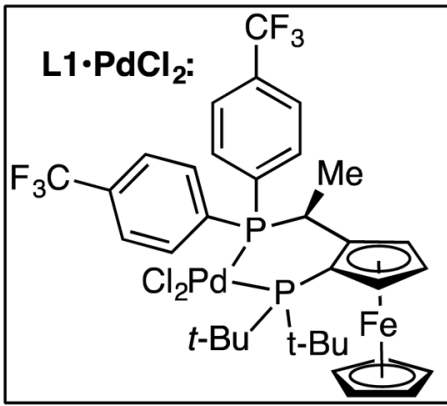

- simple starting materials

- regioselective

- enantiomerically enriched

Scheme 1.

Sequential Cross-Coupling Approach to the Asymmetric Construction of Quaternary Carbon Stereoenters 


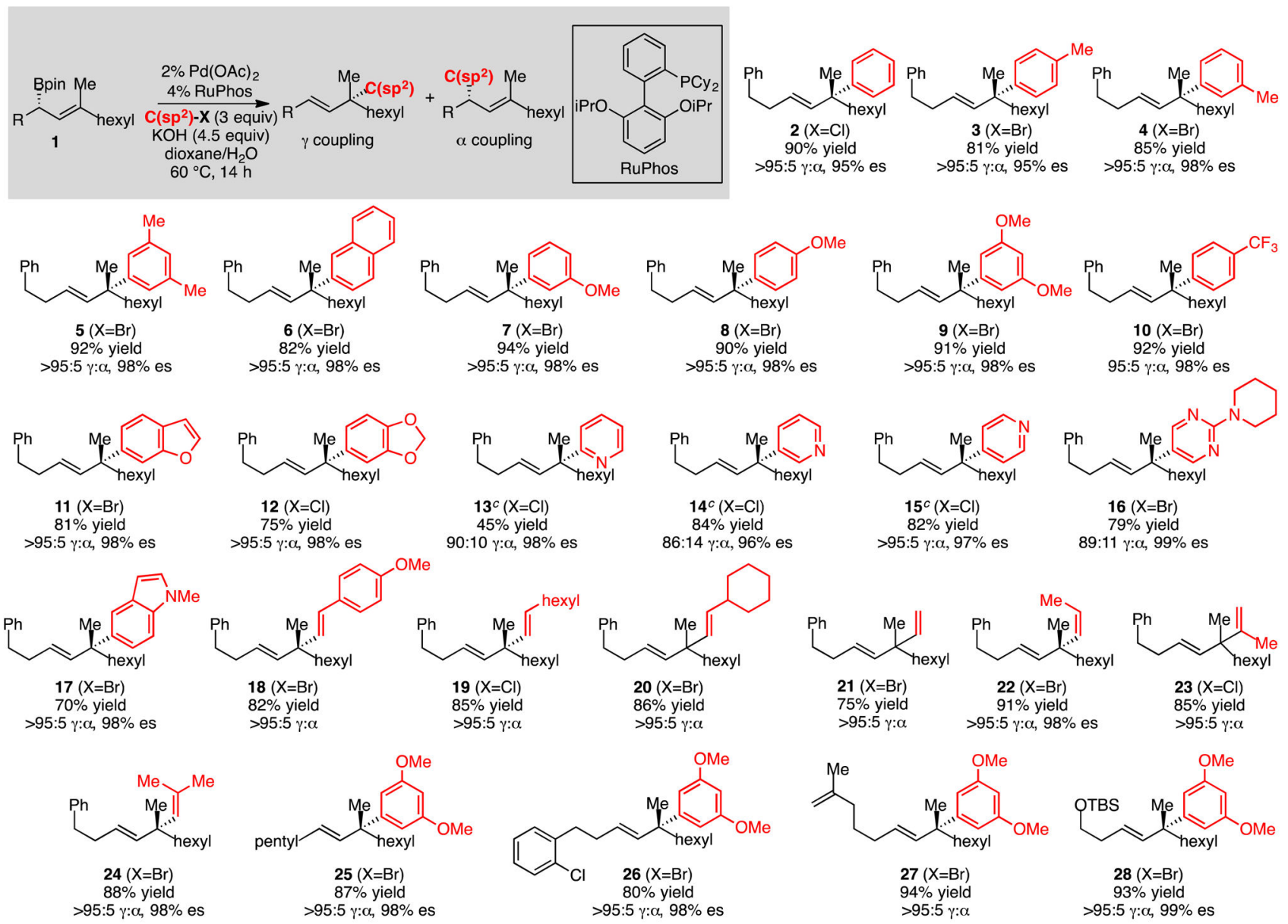

Scheme 2.

Survey of Substrates for Pd/RuPhos Catalyzed Cross-Coupling of Allylboronates and Electrophiles. ${ }^{a}$

${ }^{a}$ Reactions conducted on $0.1 \mathrm{mmol}$ scale at $60{ }^{\circ} \mathrm{C}$ for aryl electrophiles or $50{ }^{\circ} \mathrm{C}$ for alkenyl electrophiles. ${ }^{b}$ Yield is isolated yield of purified material. Regioisomer ratios were determined by ${ }^{1} \mathrm{H}$ NMR analysis, enantiospecificity determined by chiral SFC analysis. ${ }^{c}$ Employed 3.0 equiv chloropyridine $\bullet \mathrm{HCl}$ and 7.5 equiv $\mathrm{KOH}$. 

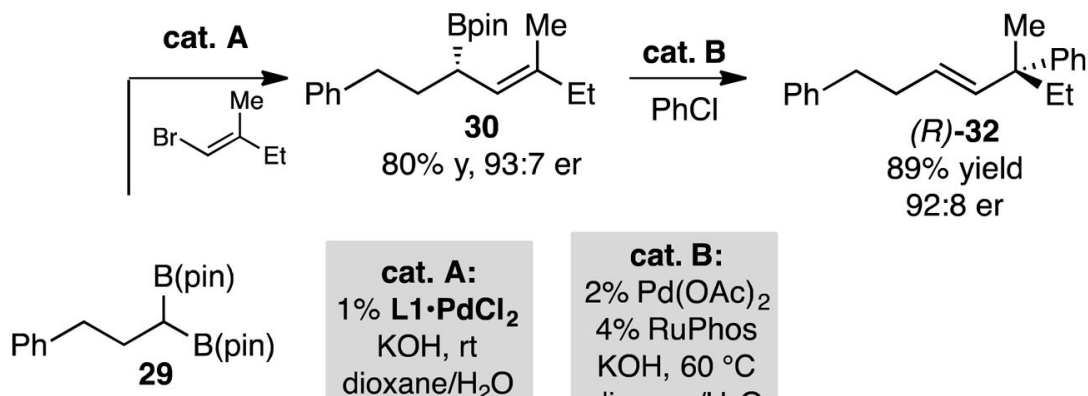

cat. A: $1 \%$ L1 $\cdot \mathbf{P d C l}_{2}$ $\mathrm{KOH}, \mathrm{rt}$ dioxane/ $/ \mathrm{H}_{2} \mathrm{O}$

cat. B:

$2 \% \mathrm{Pd}(\mathrm{OAc})_{2}$

4\% RuPhos

$\mathrm{KOH}, 60^{\circ} \mathrm{C}$

dioxane/ $\mathrm{H}_{2} \mathrm{O}$
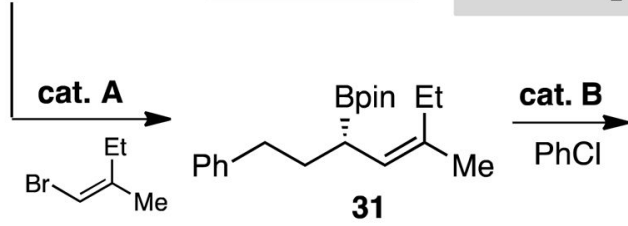

$80 \%$ y, $95: 5$ er

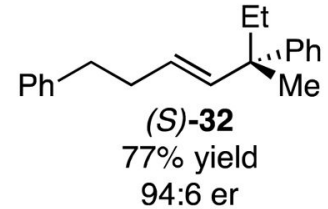

Scheme 3.

Sequential Cross-Coupling to Furnish Enantiomeric Products from the Same Enantiomer of Catalyst. 
<smiles>BrC(Br)CCc1ccccc1</smiles>

29<smiles>[B]C(Br)CCCP</smiles>

29<smiles>BrC(Br)CCc1ccccc1</smiles>

29<smiles>CC(=CBr)[Mg][Na]</smiles>

33

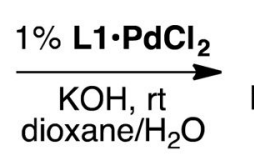

then $\mathrm{PhBr}$ ( 3 equiv)

$60^{\circ} \mathrm{C}$

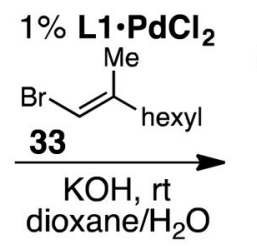

$1 \% \mathbf{L} 1 \cdot \mathbf{P d C l}_{2}$

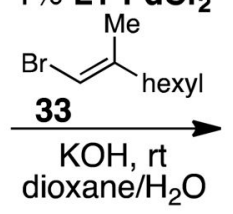

$2 \% \operatorname{Pd}(\mathrm{OAc})$

$4 \%$ RuPhos

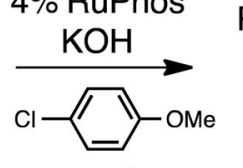

$60^{\circ} \mathrm{C}$

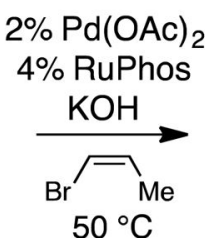<smiles>CC(=C[C@@H](Br)CCc1ccccc1)C(=O)O</smiles>

34

$66 \%$ yield

(with 17\% 2, 9\% elim.)<smiles>C/C=C\[C@](C)(/C=C/CCc1ccccc1)[18O][Mg]</smiles>

Scheme 4.

Single-Flask Sequential Cross-Couplings 
<smiles>CCC(Br)C(Br)Br</smiles>

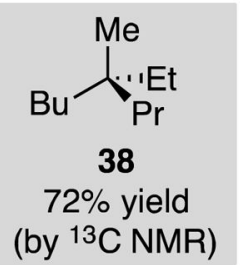

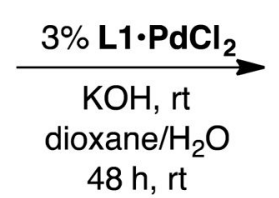

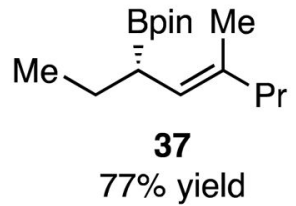

1. $2 \% \mathrm{Pd}(\mathrm{OAc})_{2} 91: 9$ er

$4 \%$ RuPhos

vinyl bromide

$\mathrm{KOH}$, dioxane/ $\mathrm{H}_{2} \mathrm{O}$ $50^{\circ} \mathrm{C}, 14 \mathrm{~h}$

2. $10 \% \mathrm{Pd} / \mathrm{C}$

$\mathrm{H}_{2}$, EtOH

Scheme 5.

Catalytic Synthesis of Chiral Hydrocarbon 38. 


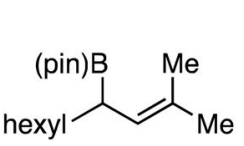

39

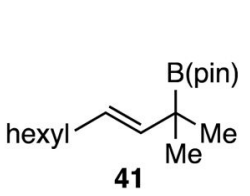

41
$2 \% \mathrm{Pd}(\mathrm{OAc})_{2}$

4\% RuPhos

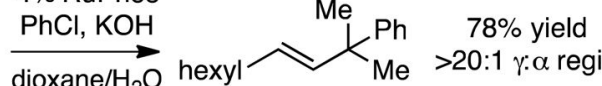

$60^{\circ} \mathrm{C}, 14 \mathrm{~h} \quad 40$

$2 \% \mathrm{Pd}(\mathrm{OAc})_{2}$

4\% RuPhos

$\mathrm{PhCl}, \mathrm{KOH} \quad \mathrm{Ph} \mathrm{Me} \quad 78 \%$ yield

$\underset{\text { dioxane } / \mathrm{H}_{2} \mathrm{O}}{\longrightarrow}$ hexyl $>20: 1 \gamma$ : $\alpha$ regio

42

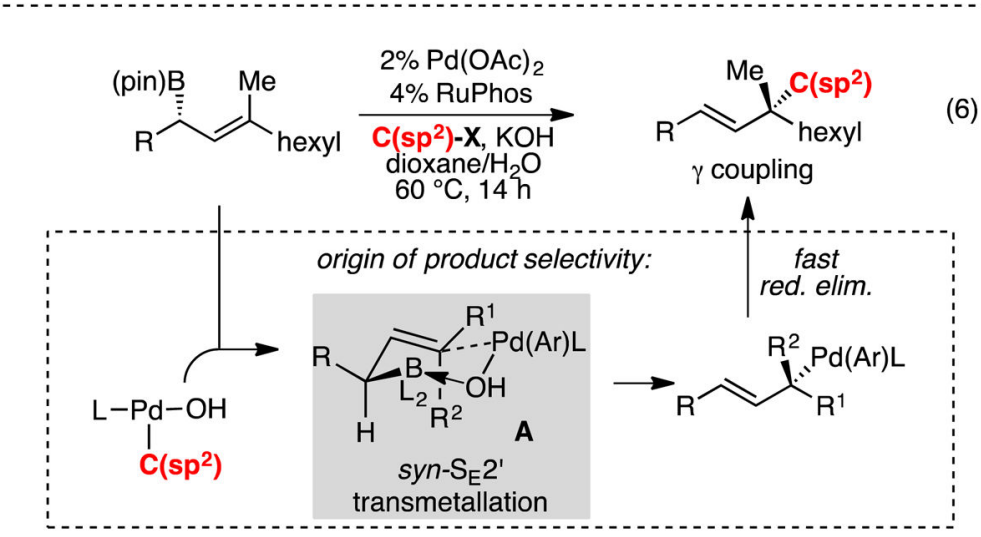

Scheme 6.

Mechanistic Experiments and the Origin of Regioselectivity 